\title{
Targeting tumor microenvironment: Metformin suppresses IL-22 induced hepatocellular carcinoma by upregulating Hippo signaling pathway
}

Dong Zhao ( $\sim$ dongzhaoltx@gmail.com )

Shanghai Jiao Tong University School of Medicine Affiliated Renji Hospital

\section{Tao Zhou}

Shanghai Jiao Tong University School of Medicine Affiliated Renji Hospital

Yi Luo

Shanghai Jiao Tong University School of Medicine Affiliated Renji Hospital

Chenchen Wang

Shanghai Jiao Tong University School of Medicine Affiliated Renji Hospital

\section{Dongwei Xu}

Shanghai Jiao Tong University School of Medicine Affiliated Renji Hospital

Chengpeng Zhong

Shanghai Jiao Tong University School of Medicine Affiliated Renji Hospital

Jianjun Zhang

Shanghai Jiao Tong University School of Medicine Affiliated Renji Hospital

\section{Qiang Xia}

Shanghai Jiao Tong University School of Medicine Affiliated Renji Hospital

\section{Research}

Keywords: metformin, IL-22, HCC, Hippo signal pathway

Posted Date: June 22nd, 2020

DOI: https://doi.org/10.21203/rs.3.rs-35094/v1

License: (c) (1) This work is licensed under a Creative Commons Attribution 4.0 International License.

Read Full License 


\section{Abstract}

\section{Background}

Epidemiological studies have shown direct associations between type 2 diabetes and the risk of cancers. Accumulating evidence indicates that metformin is profoundly implicated in preventing tumor development. However, the exact mechanism underlying the anti-tumor effects of metformin in hepatocellular carcinoma (HCC) is still not clear.

\section{Methods}

In this study, we investigated the effects of metformin on a mouse hepatocellular carcinoma (HCC) model and interleukin-22 (IL-22)-associated carcinogenesis in vitro.

Results

We found that metformin significantly suppressed the incidence and tumor burden of HCC in the diethylnitrosamine (DEN)-induced HCC mouse model. As expected, expression of IL-22, an important factor involved in HCC progression, was markedly reduced by metformin. Treatment of HCC cells with metformin inhibited IL-22 induced cell proliferation, migration and invasion, and promoted cell apoptosis. Furthermore, ectopic expression of IL-22 makes HCC more aggressive whereas metformin largely compromised it in vitro and in vivo. Mechanistically, the whole transcriptome analysis and functional analysis revealed that Hippo signaling pathway was involved in the anti-tumor ability of metformin. Consistent with this, metformin directly activated Mst1/2, phosphorylated YAP1 in vitro. After blocking Hippo pathway by XMU-MP-1, the inhibitor of MST1/2, the inhibitory effects by metformin were dramatically attenuated as shown by in vitro study.

\section{Conclusions}

Collectively, our findings illuminate a new regulatory mechanism, metformin activates Hippo signaling pathway to regulate IL-22 mediated HCC progression and provide new insights into its tumor-suppressive roles.

\section{Background}

Hepatocellular carcinoma (HCC) is the fifth most life-threatening cancer worldwide[1]. It is characterized by a high degree of malignancy, poor prognosis, high recurrence, and metastasis rate. There are about 750,000 new cases every year in the world, and about half of them are concentrated in China. Although there are many treatment options for HCC in the clinic, the prognosis of HCC patients is still unsatisfactory. Therefore, deciphering the underlying mechanisms of HCC progression might develop more precise and effective treatment strategies. 
Accumulated epidemiological studies have shown that there is a correlation between the incidence of diabetes and many types of human cancers[2-4]. Emerging data suggest that metformin can not only effectively lower blood sugar levels and improve insulin resistance, but also reduce the incidence of tumors, inhibits tumor growth and enhances the role of chemotherapy drugs [5,6]. Many studies have documented the potential mechanism of the beneficial roles of metformin in cancers. For example, metformin can activate AMPK to inhibit mTOR activity and global protein synthesis in many cancer cells [7]. Besides, metformin can selectively target cancer stem cells and act along with chemotherapy to inhibit tumor growth and prolong remission [8]. However, the anti-tumor effects of metformin in cancers are still controversial.

During the development and progression of HCC, the microenvironment in which tumor cells are located plays an essential role and affects the therapeutic effect. Different from other tumors, the tumor microenvironment of $\mathrm{HCC}$ is an extremely complex system consisting of many cell types, extracellular matrix, various cytokines and other chemical molecules[9]. These factors synergistically promote the invasion, proliferation, and metastasis of tumor cells. Among the diverse microenvironmental components, interleukin-22 (IL-22) plays an important role. IL-22, secreted by active T lymphocytes, belongs to the interleukin IL-10 family and acts primarily on epithelial cells expressing the IL-22 receptor (IL-22R1) [10]. It's believed to be involved in the development of liver diseases. During the process of liver inflammation, the expression of IL-22 is elevated and mediates the repair of liver tissue, thereby attenuating the inflammation-induced damage effect [11]. Also, IL-22 expression is significantly increased in tumor infiltrated leukocytes (TILs) and peripheral serum of HCC patients [12]. In the diethyl-nitrosamine (DEN)-induced HCC mouse model, the incidence of HCC in IL-22 knockout mice is significantly lower than that in the wild type mice [13].

In this study, we aimed to determine anti-tumor mechanism of metformin further, and explore whether it can mediate tumor-suppressive effects in the context of IL-22.

\section{Methods}

\section{Animals}

C57BL/6 male mice weighing 25-30 gr were purchased from SLRC Laboratory Animal Company (Shanghai, China) and bred in a standard vivarium with 12-h light/dark cycles and free access to food and water. Another 24 immunosuppressed adult male mice (BALB/c nude) weighing 20-35 g were purchased from SLRC Laboratory Animal Company (Shanghai, China) and kept in mini-isolator cages in groups of eight animals per cage, with positive pressure at $22^{\circ} \mathrm{C}$, light/dark cycle of $14 / 10$ hours and free access to proper water and food. All the animals were kept under germfree conditions. Mouse experiments were conducted following the National Guidelines for the Care and Use of Laboratory Animals, and the study was approved by local Institutional Animal Care and Use Committee.

\section{DEN-induced mouse HCC model and treatment}


A total of 30 male mice were used. At the age of 14 days, mice were injected with a single i.p. injection of the carcinogen DEN ( $5 \mathrm{mg} / \mathrm{kg}$ of body weight) for the induction of HCC. Among them, fifteen were further treated 2 weeks after DEN injection with metformin $(50 \mathrm{mg} / \mathrm{kg}) 3$ times a week until the end of the experiment. Saline as a vehicle $(100 \mu \mathrm{l})$ was given to the untreated HCC animals three times a week $(n=15)$. The mice were killed 48 weeks later with blood and liver tissue collected.

\section{Subcutaneous xenograft experiment}

A total of $2 \times 10^{6}$ SMMC-7721 cells stably transfected with IL-22 (PCDH-IL-22) were injected subcutaneously into the right flank of the nude mice to establish xenograft model. PCDH-NC (the pcDNA3 vector control DNA transfected HCC cells) injected mice were used as control. After 1 week of injection, metformin $(50 \mathrm{mg} / \mathrm{kg}$ ) was given 3 times a week until the end of the experiment (Met group, $\mathrm{n}=8)$. Saline as a vehicle $(100 \mu \mathrm{l})$ was given to untreated $\mathrm{HCC}$ animals three times a week ( 8 mice for each group). The mice were killed 8 weeks later with liver and tumor tissue collected. The formula estimated tumor volume $=($ Tumor length $) \times($ Tumor width $) 2 \times(\pi / 6)$.

Enzyme-linked immunosorbent assay (ELISA), Quantitative real-time PCR, Cell culture and reagents ,IL-22 Expression Vector, Cell transfection, Cell viability assay

Cell apoptosis assay, Cell migration and invasion assay, Immunohistochemical analysis, TUNEL staining, RNA-seq analysis, Western blotting analysis

Detailed information is provided in the Supplementary Materials and Methods.

\section{Statistical analysis}

All experiments were conducted independently for three times, and representative examples are shown. Data are reported as the means \pm SD. Statistical analyses were performed using SPSS 16.0 (SPSS, Chicago, IL, USA) or Prism 5.0 (GraphPad, La Jolla, CA, USA). The differences between groups were analyzed using Student's t-test or one-way ANOVA with Dunnett's multiple comparisons. $P<0.05$ was considered to be statistically significant. ${ }^{*} P<0.05,{ }^{*} \mathrm{P}<0.01$, and $* \star * P<0.001$.

\section{Results}

\section{Metformin inhibits tumorigenesis via decreasing IL-22 secretion in DEN-induced HCC mouse model}

By generating a classic DEN-induced murine HCC model, we evaluated the anti-tumor effect of metformin in HCC. The mice were given metformin $(200 \mathrm{mg} / \mathrm{kg})$ three times a week, and the control group was given normal saline. As a result, the tumor burden of primary HCC was significantly attenuated at 48 weeks (Fig. 1A). The liver and lung tissue metastases were also significantly down-regulated by metformin (Fig. 1B). The mean numbers of metastatic lung lesions in met-treated mice $(9 \pm 1.21)$ were significantly decreased compared with untreated $\triangle 14.13 \pm 1.79 \unrhd \mathrm{p}=0.03 \rrbracket$. Similar findings were observed with the mean number of metastatic liver lesions, which were $7 \pm 4.41$ for met-treated group, $12.88 \pm 5.87$ for untreated, 
respectively $(p=0.029)$. The immunohistochemistry and Integrated optical density (IOD) demonstrated that the protein levels of IL-22 in tumors from met-treated group were lower than those from untreated $(p=0.032$, Fig. $1 \mathrm{C})$. Consistently, metformin treatment also led to approximately half reduction of the

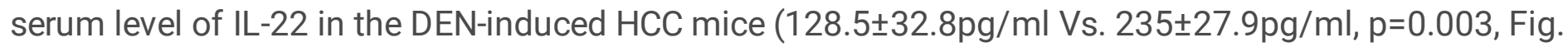
1D). Then we analyzed the mRNA level of IL-22 in the normal liver and tumor tissues. The real-time PCR result showed that IL-22 mRNA expression was markedly decreased in metformin-treated tumor tissues compared with that in tumors isolated from untreated mice ( $p=0.002$, Fig.1E). Collectively, the phenomena are in accordance with our previous findings, which suggest metformin might inhibit HCC tumorigenesis by down-regulating of IL-22.

\section{Metformin weakens IL-22 driven HCC aggressiveness in vitro}

Next, we aimed to uncover the anti-tumor roles of metformin in the presence or absence of IL-22 by in vitro IL-22 gain-of-function studies using SMMC-7721 and 97H cells. The overexpression efficiency was certified by real-time qPCR analysis ( $p<0.001$, Fig. $2 A)$. The CCK-8 experiment showed that the cell viabilities of SMMC-7721 and $97 \mathrm{H}$ cells were largely restored by IL-22 while impaired by metformin treatment (Fig. 2B). Of note, the Annexin V/PI apoptosis assay showed that metformin treatment induced the increased cell apoptosis compromised by overexpression of IL-22 in both SMMC-7721 and 97H cells (Fig. 2C). Furthermore, the transwell boyden model was used to determine the role of metformin on the malignant phenotypes of HCC cells. As anticipated, the migratory and invasive capacity of SMMC-7721 and $97 \mathrm{H}$ cells were significantly increased by IL-22 overexpression, while metformin treatment overcame the growth disadvantage (Fig. 2D,2E).

\section{Metformin represses IL-22 induced HCC in vivo}

To further testify the in vitro effect of metformin on HCC, we generated a subcutaneous xenograft model with IL-22 transfected SMMC-7721 cells. As a result, the tumor burden of the xenograft formed from IL-22 transfected cells were significantly relieved by metformin treatment (Fig.3A-C). The mean tumor weight in the metformin treatment group $(0.22 \pm 0.04)$ was dramatically decreased compared to that from IL-22 transfected group $(0.52 \pm 0.32, p=0.0021$, Fig. 3B). By immunohistochemical analysis, we found that metformin-treated tumor tissues had lower proliferative and invasive ability as revealed by decreased staining of the proliferation index Ki67 and the invasive marker MMP9 (Fig. 3D-E). Consistently, IL-22 overexpression effectively suppressed the staining of the apoptosis rate compared to the control while metformin treatment restrained the disadvantage as revealed by TUNEL staining (Fig. 3F). Taken together, these data suggest that metformin inhibits IL-22 related HCC oncogenic activities by inhibiting cell growth and accelerating apoptosis in vivo.

\section{Hippo signaling is revealed high in vivo by transcriptional analysis}

To characterize the mechanism by which metformin inhibits HCC progression, RNAseq analysis was performed with liver tissues from DEN induced HCC model. As a result, many differentially expressed genes were identified (Fig. 4A, B). By Kyoto Encyclopedia of Genes and Genomes (KEGG) annotation, we 
found that Hippo signaling pathway was enriched with high confidence in metformin-treated group (Fig. 4C). To further explore whether metformin affects Hippo signaling, we detected the mRNA expression of several downstream target genes of YES-associated protein (YAP) in metformin treated and untreated group by real-time qPCR. The result showed that expression level of connective tissue growth factor (CTGF), ankyrin repeat domain 1 (ANKRD1), cysteine-rich angiogenic inducer 61 (CYR61), indoleamine 2,3-dioxygenase 1 (ID01), replication timing regulatory factor 1 (RIF1), and DExD/H-Box helicase 60 (DDX60) were significantly reduced by metformin(Fig. 4D).

\section{Hippo pathway regulated by metformin contributes to its HCC suppressive effect}

Finally, we examined the transcriptional findings in vitro studies. The levels of Mst1, Mst2(Mst1/2), YAP and pYAP were tested by western blotting. Metformin treatment $(400 \mu \mathrm{M}, 48$ hours $)$ induced strong activation of Hippo signaling in SMMC-7721 cells stably transfected with IL-22 as shown by markedly expression of Mst1/2 and pYAP (Fig.5A). Consistently, low mRNA levels of CTGF, IOD1 and DDX60 were also observed (Fig.5B). We next explored if the anti-malignancy potential of metformin is Hippo independent. In the presence of MST1/2 inhibitor, XMU-MP-1, we silenced Hippo pathway and examined the biological behavior of stably transfected SMMC-7721 cells. As shown in Fig.5C, the apoptosis induced by metformin was abrogated when Hippo signaling blocked. Cell viabilities restoration were also detected following inhibition of Hippo pathway (Fig.5D). XMU-MP-1 also significantly compromised the anti-migratory (Fig. 5E) and anti-invasive (Fig. 5F) effect mediated by metformin. Taken together, these data provide the potential mechanism of metformin action for HCC progression by which it can interfere with cancer prevention Hippo signaling pathway.

\section{Discussion}

The biology of tumors can only be understood by studying variant cell types within the tumor microenvironment (TME). The heterogeneity of tumors is based not only on the genomic profile but also on their microenvironment composition [14]. The microenvironment actively regulates tumor initiation, its progression, metastasis, and therapy response [15]. Hepatocarcinogenesis is a multifactorial process. Most HCC cases in western countries are associated with nonalcoholic steatohepatitis (NASH), alcohol abuse, hepatitis $\mathrm{C}$ virus (HCV), while in China, most with chronic infection with hepatitis $B$ virus (HBV) inducing an inflammatory process followed by regeneration. Persistent concurrent regeneration and hepatic injury could produce an environment that eventually leads to the formation of hypoxia and inflammation, which are crucial features of $\mathrm{HCC}[16]$. The inflammatory microenvironment facilitates the transformation of normal liver cells such as hepatocytes, immune, and stellate cells by providing a suitable environment for the development and progression of a tumor. Sustained inflammation followed by a continuous activation of immune cells can damage the DNA and bring about a neoplastic transformation. IL-22, one of the main T helper cell(Th17)-derived cytokines, has been linked as a vital element that allows tumorigenesis. Overexpression of IL-22 has been observed in several human tumors, such as liver, breast and prostate cancers. It has the capacity of prompting cell differentiation and proliferation, and, in mice models, some reports suggest that IL-22 favors tumor metastasis in lung, colon 
cancers and non-melanoma skin cancer[17]. In humans gastric, colorectal and pancreatic cancer, elevated expression of IL-22 and IL-22 receptor were reported to correlate with disease progression and poor overall survival[ $[18,19]$. It has also been observed that patients with liver fibrosis and advanced cirrhosis present high serum levels of IL-22. Consistent with our previous study, we further verified the inhibitory effect of metformin on HCC by using DEN-induced mouse model. This model mimics aspects of liver injury, hepatitis and fibrosis, which all are the basis of human HCC[20]. Despite current findings, transgenic murine models with IL-22 overexpression and knockout are warranted to investigate to achieve an even convincing agreement.

Once a tumor is established, various cytokines can be recruited from a distant place of the same organ or peripheral tissues into the TME. The persistent inflammatory milieu not only promotes tumor development but also accelerates tumor progression, stimulates invasion, angiogenesis, and metastasis through the release of several mediators. Our data indicate that IL-22 is one of these crucial mediators for tumor progression and metastasis. The results are in accordance with Jiang et.al [13]which shown IL-22 had a prominent effect on tumor cell survival, proliferation, invasion, metastasis, as well as malignancy transformation from chronic hepatitis. It's easy to understand that any changes in the microenvironment could support the development of HCC, and the complexity of TME and therapeutic failures may be explained, in some degree, by alterations of components of the TME. Metformin was introduced into HCC treatment in our study and had shown to restrain IL-22 related tumorigenesis and progression both in vitro and in vivo.

However, HCC microenvironment is composed of numerous tumoral and non-tumoral cell types, and cytokines and other components that are in continuous interaction and communication with each other. Their interactions make an important contribution to tumor progression by modulating tumor cell properties. This scheme is far more complex and requires orchestrated regulation. In addition, functions of numerous cytokines secreted by diverse immune cells upon certain stimuli may overlap. The intricacy of the system means, on the other side, that effect of metformin on one single cytokine within tumor tissue extremely depends on the context of the individual microenvironment and is hard to predict. The major challenge of future studies remains to predict the role for an individual response in any given microenvironment.

MHCC97-H and SMMC-7721 are two types of human HCC cell lines with varying biological behaviors. MHCC97-H cell is characterized by larger cell volume and a highly invasive phenotype[21].SMMC-7721 cell, originated from a Chinese HCC patient, is widely used in the HCC research concerning anticancer effects and mechanisms of various anti-cancer drugs[22]. In the present study, IL-22 promoted cell proliferation, survival, metastasis and invasion compared with control cell lines, whereas the disadvantage was significantly undermined by metformin treatment. This result was confirmed in vivo using subcutaneous xenograft model. The underlying mechanism probably involved Hippo pathway. However, it should be noted that heterogeneity between HCC cells need to be taken into account. Further studies with more different HCC cells or tissues can be more informative. 
The Hippo signaling pathway, an highly conserved signaling pathway regulated by Mst $1 / 2$, controls organ size, tissue regeneration, as well as tumor progression through the regulation of cell apoptosis and proliferation[23]. YAP, a protein that acts as a transcriptional regulator by activating the transcription of genes involved in cell apoptosis and proliferation, is the main downstream effectors of the Hippo signaling pathway[24].CYR61 and CTGF, direct targets of YAP, belong to extracellular matrix -associated signaling protein of the $\mathrm{CCN}$ family which are capable of regulating a broad range of cellular activities, such as cell adhesion, proliferation, senescence and apoptosis[25]. Given that they play a positive role in cell proliferation, it's not surprising that these two proteins have been found to be elevated in some human cancers, including pancreatic malignancy[26], gliomas[27], prostate cancer[28] and breast cancer [29]. Abnormal activation of TAZ and YAP caused by aberrant Hippo signaling is continually observed in many human cancers [30]. Yap ectopic expressed specifically in liver can result in a significant increase in size. Remarkably, the liver reverts back to its properly size when Yap overexpression is turned off [31]. Similarly, enlargement of liver is also reported in Mst1/2 knockout mice[32]. These observations suggest that YAP/TAZ are identified as oncogenes involved in the Hippo signaling pathway. Importantly, activation of YAP as a consequence of abrogating Hippo signaling by knocking out Mst $1 / 2$ was sufficient to drive liver carcinogenesis in mice[33]. Interestingly, we found that IL-22 transfected tumor cell resulted in inactivation of Hippo signaling as shown by lower expression of Mst1/2 and afterwards phosphorylation of YAP which leads to the suppression of YAP activity. Meanwhile, metformin dramatically impaired this tumorigenesis effect. The mechanism of which metformin regulates the Hippo pathway could be attributed to activation of AMP-activated protein kinase (AMPK)[34]and this makes our results more understandable. However, it should be noted that many factors, such as proteins that determine cell adherens, tight junctions and polarity, can contribute to regulation of Hippo signaling pathway. Thus, it still remains to examine further possible mechanisms involved in the presence of metformin, on the other hand, a similar experimental design with Mst1/2 knockout mice is warranted to investigate the therapeutic potential of metformin in the prevention of HCC.

Given that TME is deeply involved in many human cancers, there is a growing enthusiasm in developing drugs that may affect it as a promising cancer treatment. The data from mouse models and cell experiments studied in this work raise the possibility that aiming at TME metformin could have desirable antitumorigenic effects, however, its clinical utility is yet to be ascertained.

\section{Conclusions}

Epidemiological studies suggest that treatment with metformin in patients with type 2 diabetes mellitus reduces the incidence of cancer at a number of tumor sites. The mechanism of action of metformin on tumor cells, however, has remained unclear. Therefore, we designed an in-depth study to try and answer some of the questions and explored potential mechanisms. We described that metformin inhibits tumor growth in the DEN-induced HCC model and an IL-22 decreased by metformin treatment as a potential mechanism. We also showed that metformin can reverse the effects induced by IL-22 overexpression, including tumor cell proliferation, cell apoptosis, and cell migration. During the mechanistic study, we 
discovered that the metformin activated the Hippo signaling pathway, and inhibiting this pathway can reverse the effect of metformin under the IL-22 overexpression background.

\section{Abbreviations}

Hepatocellular carcinoma (HCC), Metformin (Met), Interleukin-22 (IL-22), diethyl-nitrosamine (DEN), connective tissue growth factor (CTGF), ankyrin repeat domain 1 (ANKRD1), cysteine-rich angiogenic inducer 61 (CYR61), indoleamine 2,3-dioxygenase 1 (ID01), replication timing regulatory factor 1 (RIF1), and DExD/H-Box helicase 60 (DDX60), tumor microenvironment (TME)

\section{Declarations}

Ethical Approval and Consent to participate: Mouse experiments were conducted following the National Guidelines for the Care and Use of Laboratory Animals, and the study was approved by Institutional Animal Care and Use Committee of Shanghai Jiao Tong University School of Medicine Affiliated Renji Hospital.

Consent for publication: Not applicable.

Availability of supporting data:The data generated or analyzed during this study are included in this published article and its supplementary information files are available from the corresponding author on reasonable request.

Competing interests: The authors declare that they have no competing interests.

Funding Sources: This study was supported by NSFC (Natural Science Foundation of China) under Grant No. 81602487

Author contributions: D Zhao, T Zhao: Conception and design, collection and assembly of the data, data analysis and interpretation, writing, and editing. Y Luo, CC W, DW X and CP Zhong: Technical assistance during experimentation, review. JJ Zhang: Statistical analyses, review. Q Xia: Conception and design, supervision, writing, editing, and review. All authors read and approved the final manuscript.

Acknowledgements: Not applicable.

Authors' information: Not applicable.

\section{References}

1. Forner A, Reig M, Bruix J. Hepatocellular carcinoma. Lancet. 2018;391:1301-14.

2. Yki-Jarvinen H, Luukkonen PK. Diabetes, Liver Cancer, and Cirrhosis: What Next? Hepatology 2018, 68:1220-1222. 
3. Pan XF, He M, Yu C, Lv J, Guo Y, Bian Z, Yang L, Chen Y, Wu T, Chen Z, et al. Type 2 Diabetes and Risk of Incident Cancer in China: A Prospective Study Among 0.5 Million Chinese Adults. Am J Epidemiol. 2018;187:1380-91.

4. Park Y, Colditz GA. Diabetes and adiposity: a heavy load for cancer. Lancet Diabetes Endocrinol. 2018;6:82-3.

5. Lord SR, Cheng WC, Liu D, Gaude E, Haider S, Metcalf T, Patel N, Teoh EJ, Gleeson F, Bradley K, et al. Integrated Pharmacodynamic Analysis Identifies Two Metabolic Adaption Pathways to Metformin in Breast Cancer. Cell Metab. 2018;28:679-88.e674.

6. Liu Q, Tong D, Liu G, Gao J, Wang LA, Xu J, Yang X, Xie Q, Huang Y, Pang J, et al. Metformin Inhibits Prostate Cancer Progression by Targeting Tumor-Associated Inflammatory Infiltration. Clin Cancer Res. 2018;24:5622-34.

7. Howell JJ, Hellberg K, Turner M, Talbott G, Kolar MJ, Ross DS, Hoxhaj G, Saghatelian A, Shaw RJ, Manning BD. Metformin Inhibits Hepatic mTORC1 Signaling via Dose-Dependent Mechanisms Involving AMPK and the TSC Complex. Cell Metab. 2017;25:463-71.

8. Hirsch HA, lliopoulos D, Tsichlis PN, Struhl K. Metformin selectively targets cancer stem cells, and acts together with chemotherapy to block tumor growth and prolong remission. Cancer Res. 2009;69:7507-11.

9. Sia D, Jiao Y, Martinez-Quetglas I, Kuchuk O, Villacorta-Martin C, Castro de Moura M, Putra J, Camprecios G, Bassaganyas L, Akers N, et al. Identification of an Immune-specific Class of Hepatocellular Carcinoma, Based on Molecular Features. Gastroenterology. 2017;153:812-26.

10. Hernandez P, Gronke K, Diefenbach A. A catch-22: Interleukin-22 and cancer. Eur J Immunol. 2018;48:15-31.

11. Radaeva S, Sun R, Pan HN, Hong F, Gao B. Interleukin 22 (IL-22) plays a protective role in T cellmediated murine hepatitis: IL-22 is a survival factor for hepatocytes via STAT3 activation. Hepatology. 2004;39:1332-42.

12. Waidmann O, Kronenberger B, Scheiermann P, Koberle V, Muhl H, Piiper A. Interleukin-22 serum levels are a negative prognostic indicator in patients with hepatocellular carcinoma. Hepatology. 2014;59:1207.

13. Jiang R, Tan Z, Deng L, Chen Y, Xia Y, Gao Y, Wang X, Sun B. Interleukin-22 promotes human hepatocellular carcinoma by activation of STAT3. Hepatology. 2011;54:900-9.

14. Quail DF, Joyce JA. Microenvironmental regulation of tumor progression and metastasis. Nat Med. 2013;19:1423-37.

15. Klemm F, Joyce JA. Microenvironmental regulation of therapeutic response in cancer. Trends Cell Biol. 2015;25:198-213.

16. Nishida N, Kudo M. Oncogenic Signal and Tumor Microenvironment in Hepatocellular Carcinoma. Oncology. 2017;93(Suppl 1):160-4.

17. Nardinocchi L, Sonego G, Passarelli F, Avitabile S, Scarponi C, Failla CM, Simoni S, Albanesi C, Cavani A. Interleukin-17 and interleukin-22 promote tumor progression in human nonmelanoma skin cancer. 
Eur J Immunol. 2015;45:922-31.

18. Wen Z, Liao Q, Zhao J, Hu Y, You L, Lu Z, Jia C, Wei Y, Zhao Y. High expression of interleukin-22 and its receptor predicts poor prognosis in pancreatic ductal adenocarcinoma. Ann Surg Oncol. 2014;21:125-32.

19. Wu T, Cui L, Liang Z, Liu C, Liu Y, Li J. Elevated serum IL-22 levels correlate with chemoresistant condition of colorectal cancer. Clin Immunol. 2013;147:38-9.

20. Santos NP, Colaco AA, Oliveira PA. Animal models as a tool in hepatocellular carcinoma research: A Review. Tumour Biol. 2017;39:1010428317695923.

21. Tian J, Tang ZY, Ye SL, Liu YK, Lin ZY, Chen J, Xue Q. New human hepatocellular carcinoma (HCC) cell line with highly metastatic potential (MHCC97) and its expressions of the factors associated with metastasis. Br J Cancer. 1999;81:814-21.

22. Geng CX, Zeng ZC, Wang JY. Docetaxel inhibits SMMC-7721 human hepatocellular carcinoma cells growth and induces apoptosis. World J Gastroenterol. 2003;9:696-700.

23. Yu FX, Zhao B, Guan KL. Hippo Pathway in Organ Size Control, Tissue Homeostasis, and Cancer. Cell. 2015;163:811-28.

24. Zhao B, Li L, Lei Q, Guan KL. The Hippo-YAP pathway in organ size control and tumorigenesis: an updated version. Genes Dev. 2010;24:862-74.

25. Zuo GW, Kohls CD, He BC, Chen L, Zhang W, Shi Q, Zhang BQ, Kang Q, Luo J, Luo X, et al. The CCN proteins: important signaling mediators in stem cell differentiation and tumorigenesis. Histol Histopathol. 2010;25:795-806.

26. Holloway SE, Beck AW, Girard L, Jaber MR, Barnett CC Jr, Brekken RA, Fleming JB. Increased expression of Cyr61 (CCN1) identified in peritoneal metastases from human pancreatic cancer. J Am Coll Surg. 2005;200:371-7.

27. Xie D, Yin D, Wang HJ, Liu GT, Elashoff R, Black K, Koeffler HP. Levels of expression of CYR61 and CTGF are prognostic for tumor progression and survival of individuals with gliomas. Clin Cancer Res. 2004;10:2072-81.

28. Lv H, Fan E, Sun S, Ma X, Zhang X, Han DM, Cong YS. Cyr61 is up-regulated in prostate cancer and associated with the p53 gene status. J Cell Biochem. 2009;106:738-44.

29. Jiang WG, Watkins G, Fodstad O, Douglas-Jones A, Mokbel K, Mansel RE. Differential expression of the CCN family members Cyr61, CTGF and Nov in human breast cancer. Endocr Relat Cancer. 2004;11:781-91.

30. Moroishi T, Hansen CG, Guan KL. The emerging roles of YAP and TAZ in cancer. Nat Rev Cancer. 2015;15:73-9.

31. Hong L, Li Y, Liu Q, Chen Q, Chen L, Zhou D. The Hippo Signaling Pathway in Regenerative Medicine. Methods Mol Biol. 2019;1893:353-70.

32. Song H, Mak KK, Topol L, Yun K, Hu J, Garrett L, Chen Y, Park O, Chang J, Simpson RM, et al. Mammalian Mst1 and Mst2 kinases play essential roles in organ size control and tumor 
suppression. Proc Natl Acad Sci U S A. 2010;107:1431-6.

33. Lu L, Li Y, Kim SM, Bossuyt W, Liu P, Qiu Q, Wang Y, Halder G, Finegold MJ, Lee JS, Johnson RL. Hippo signaling is a potent in vivo growth and tumor suppressor pathway in the mammalian liver. Proc Natl Acad Sci U S A. 2010;107:1437-42.

34. Mo JS, Meng Z, Kim YC, Park HW, Hansen CG, Kim S, Lim DS, Guan KL. Cellular energy stress induces AMPK-mediated regulation of YAP and the Hippo pathway. Nat Cell Biol. 2015;17:500-10.

\section{Figures}

A

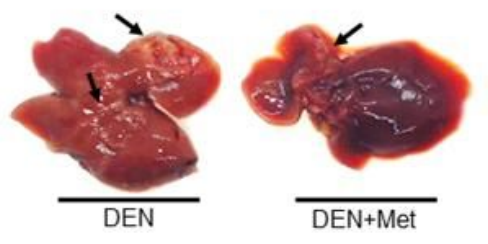

C
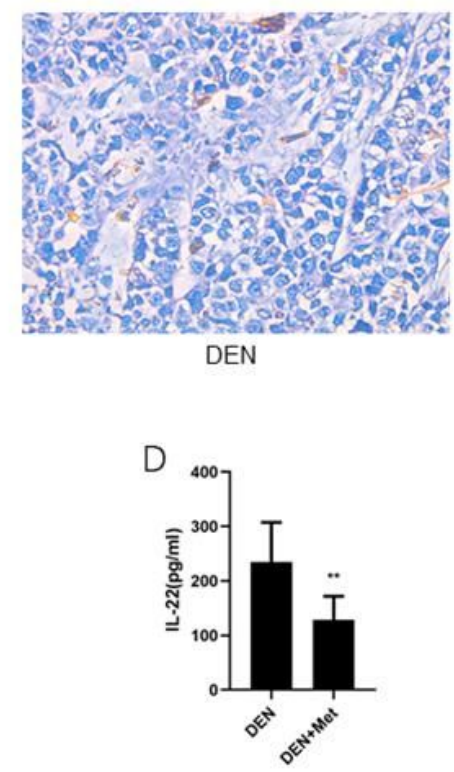

B
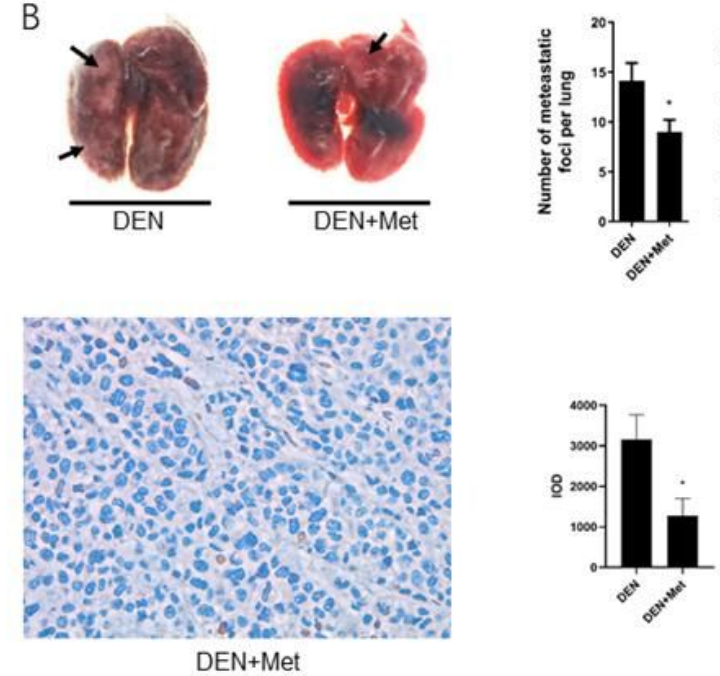

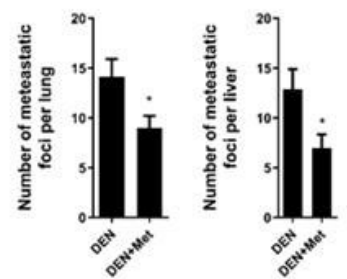

E

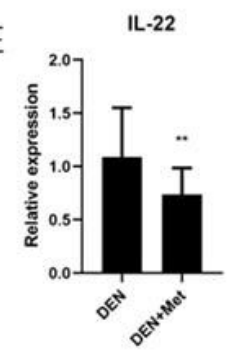

\section{Figure 1}

Metformin inhibits tumorigenesis and decreases IL-22 expression in DEN-induced HCC mouse model. Mice were treated with saline or metformin $(50 \mathrm{mg} / \mathrm{kg})$ for the indicated times as described in Material and Methods. (A)Gross morphology of representative tumors in liver and (B) lung.(C)Average number of metastatic lesions in lung and liver.(D) Expression of IL-22 protein in tumor tissues was tested using immunohistochemistry (IHC)(x200) and assessed by average IOD.(E) ELISA analysis of IL-22 in the serum.(F) IL-22 mRNA level in the tumors as detected by Real-time $\mathrm{qPCR} .{ }^{*} \mathrm{P}<0.05 ;{ }^{\star \star} \mathrm{P}<0.01$. 
A

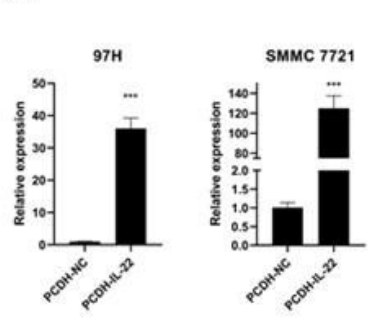

B

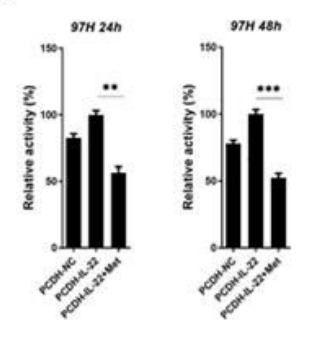

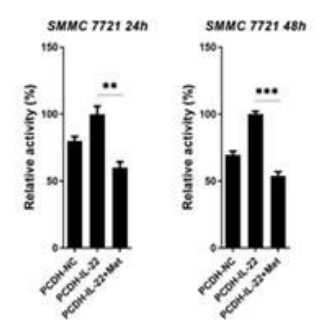

C

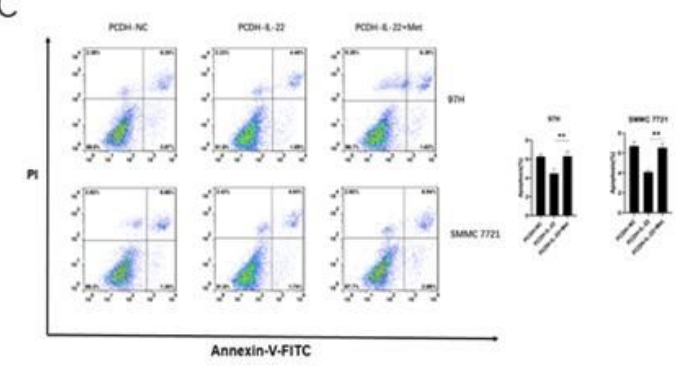

E

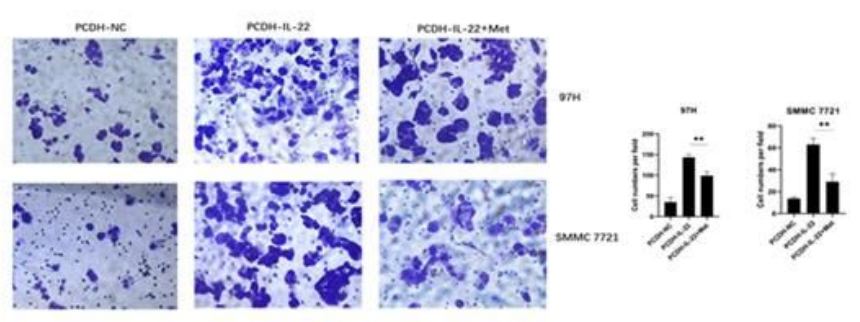

\section{Figure 2}

Metformin abrogates IL-22 overexpression HCC cells aggressiveness. MHCC97-H and SMMC-7721 cell lines were transfected with IL-22 (PCDH-IL-22) and subsequently treated with metformin(400 $\mu \mathrm{M})$. Empty vector-transfected clones (PCDH-NC) were used as control. (A) The overexpression efficiency of IL-22 in MHCC97-H and SMMC7721 cells were measured by real-time qPCR. (B) Viability of IL-22 transfected MHCC97-H and SMMC-7721 cell after metformin treatment was shown by CCK-8 assay.(C) Apoptosis rate of HCC cells in different groups as shown by Annexin V/PI assay and (D) migratory (F)as well as invasive ability. ${ }^{\mathrm{P}}<0.05 ; * \star P<0.01 ; * \star \star P<0.001$. 
A

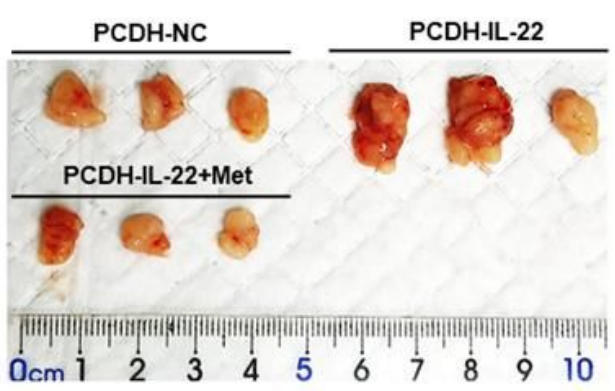

B

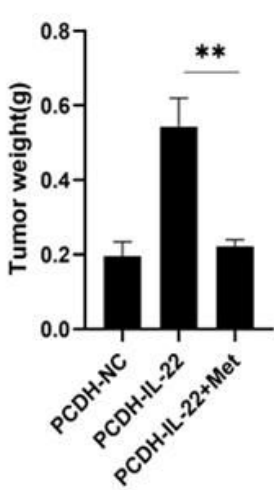

C
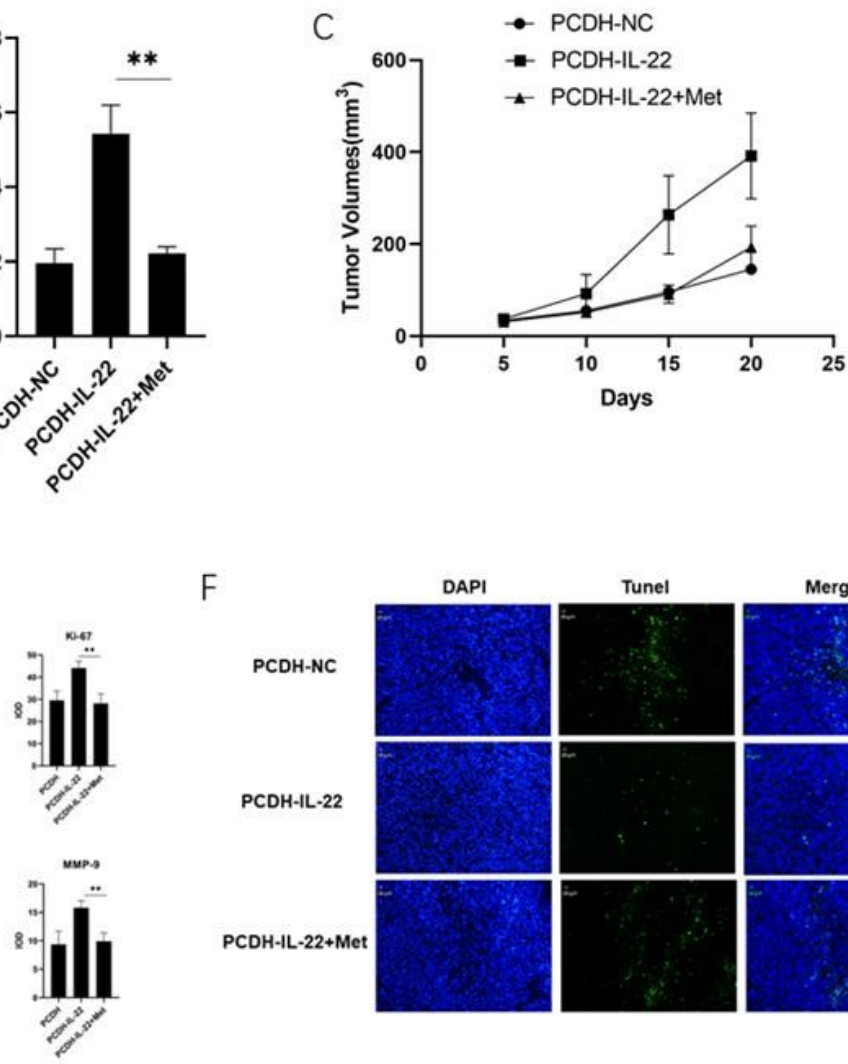

F
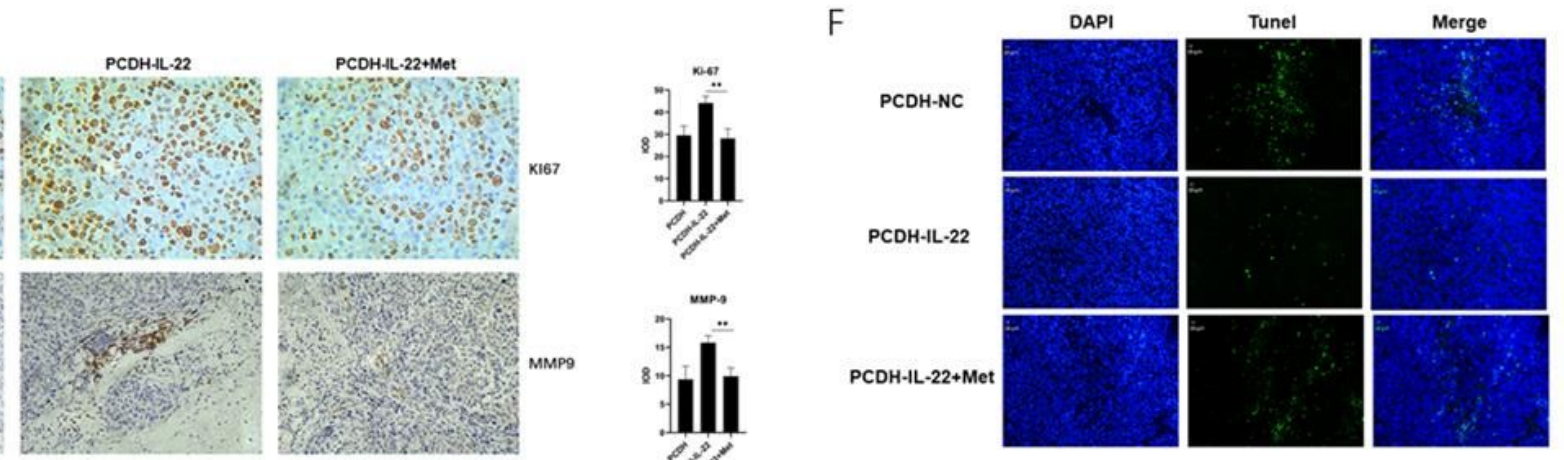

\section{Figure 3}

In vivo effect of metformin on IL-22 driven HCC. Subcutaneous xenograft model was established and treated as described in Materials and Methods. (A) Gross morphology of representative tumors, (B) mean tumor weight, (C) mean tumor volume of each group at the day the mice were euthanized. IHC analysis showed the proliferation index Ki67(D) and the invasive marker MMP9(E) in indicated groups. (F) TUNEL staining demonstrated the apoptosis rate in indicated groups. ${ }^{*} P<0.05$; ${ }^{\star *} \mathrm{P}<0.01$; ${ }^{\star \star *} \mathrm{P}<0.001$. 
A

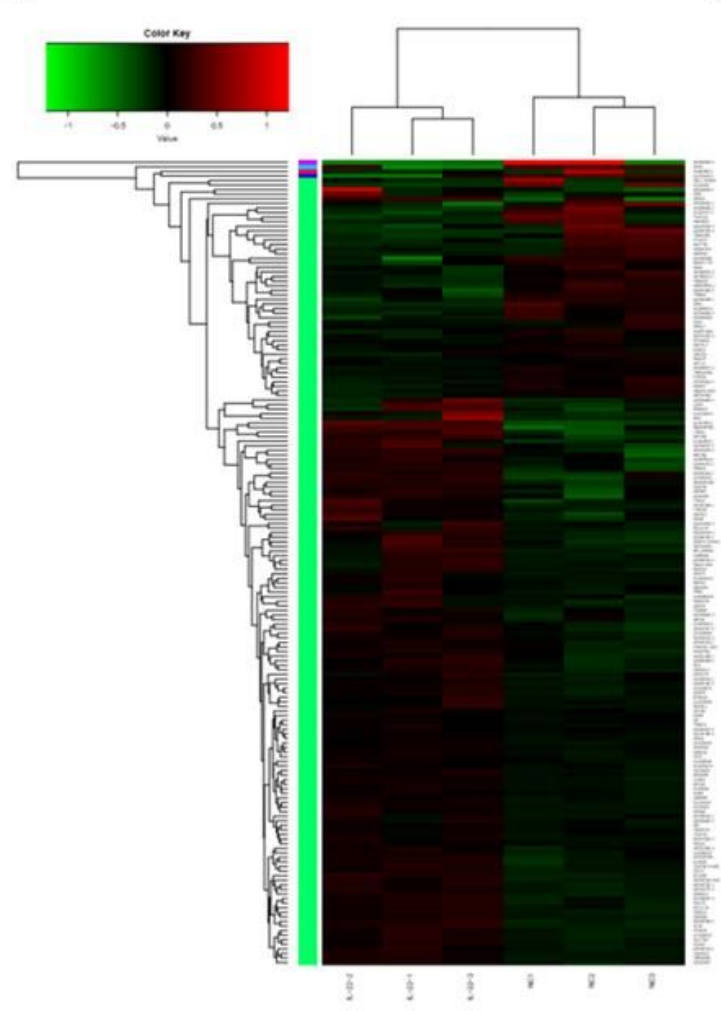

B

IL vs. NC volcano [DE: p-value It $0.05 \& \&$ llog2(Fold change)| gt 1]
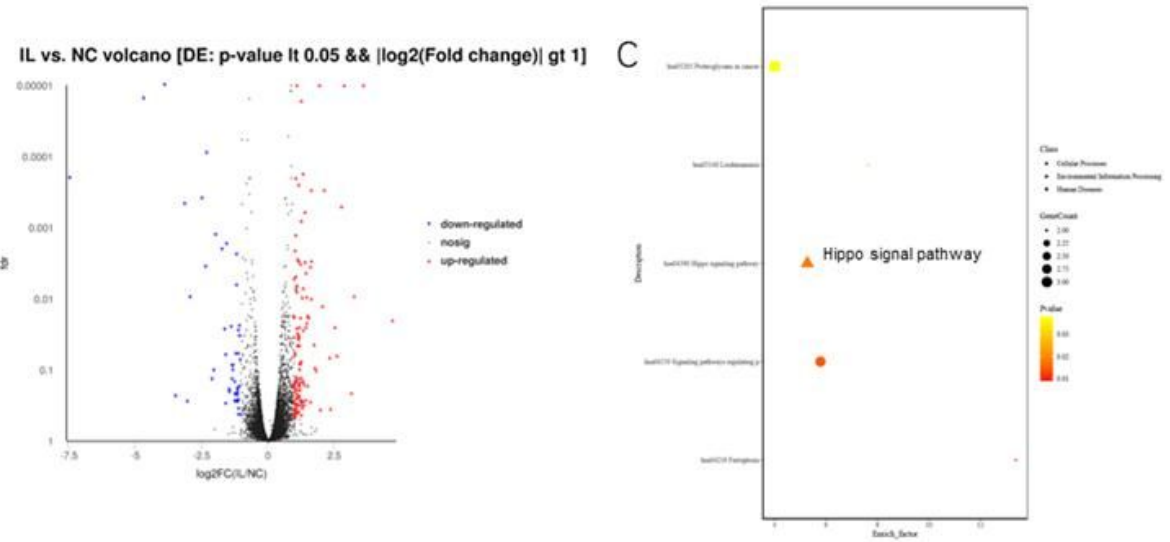

D
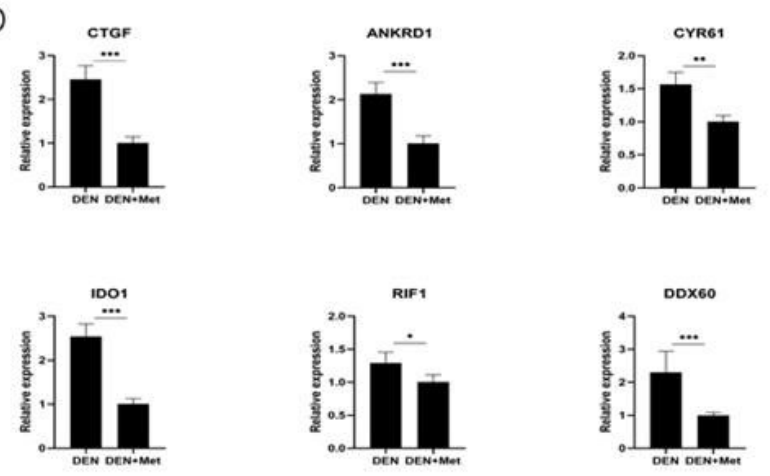

\section{Figure 4}

Transcriptional changes revealed after metformin treatment in DEN induced HCC model. Tumor tissues from treated and untreated DEN induced HCC mouse model were collected at the time of sacrifice for further RNA-seq analysis. (A) Hierarchical clustering of all differentially expressed genes (DEGs). (B) Volcano plot showed the differentially expressed genes after metformin treatment. (C) KEGG analysis highlighted that Hippo pathway was upregulated in metformin-treated group. (D) Real-time qPCR analysis of the effect of metformin treatment on the expression of the downstream target genes of Hippo signaling pathway. ${ }^{*} \mathrm{P}<0.05 ;{ }^{* *} \mathrm{P}<0.01 ; * \star * \mathrm{P}<0.001$. 

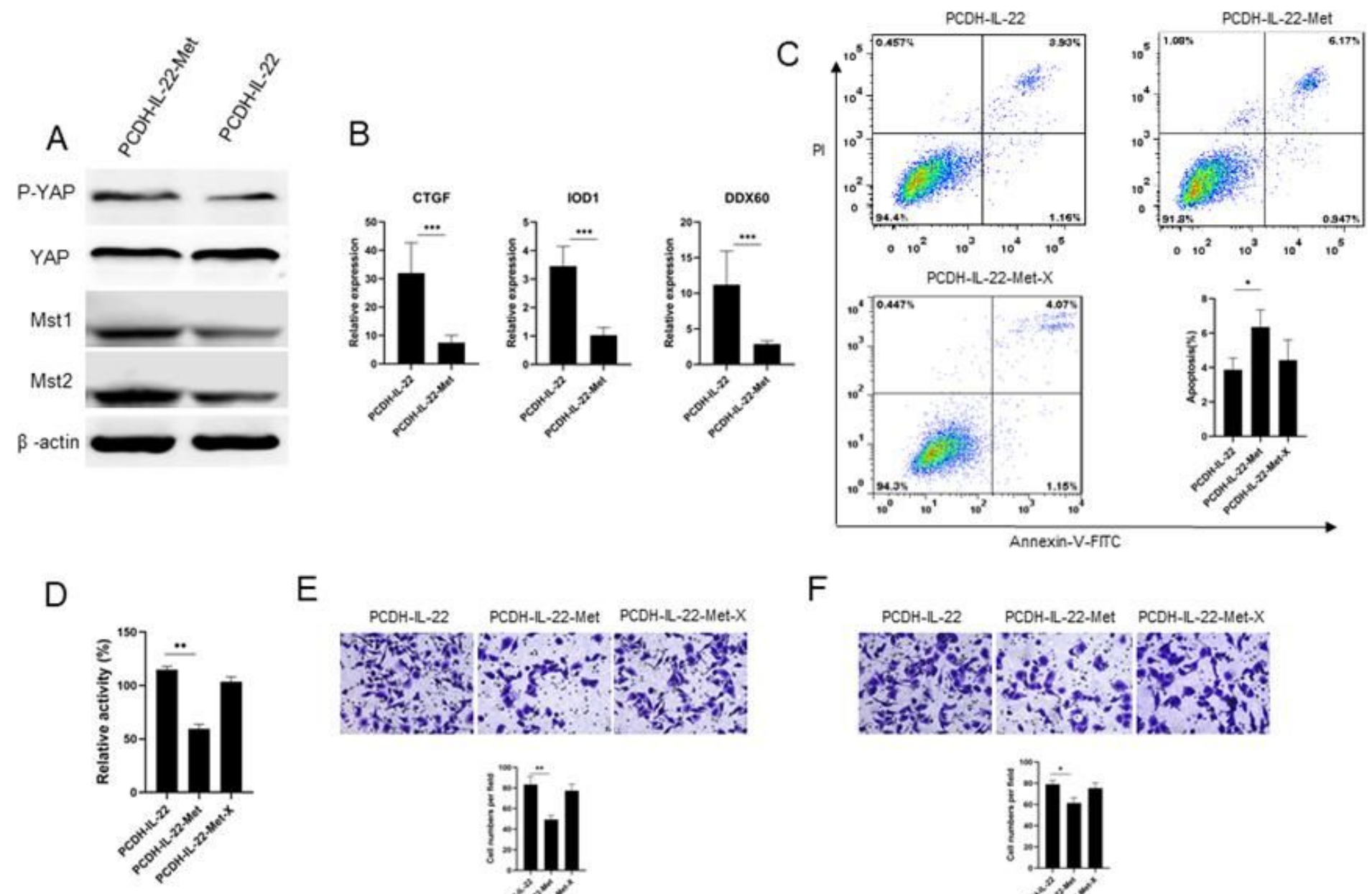

$\mathrm{E}$

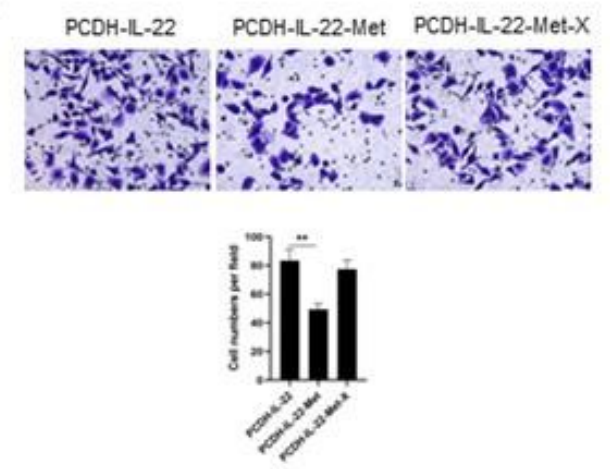

$\mathrm{F}$

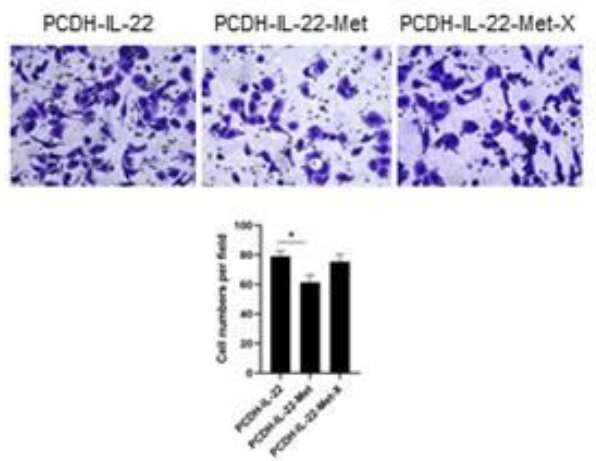

Figure 5

Metformin activates Hippo signaling pathway to inhibit IL-22 induced HCC malignancies. IL-22 transfected SMMC-7721 cells were treated with metformin $(400 \mu \mathrm{M})$ as indicated.(A) Western blot showing the protein levels of p-YAP,YAP and Mst1/2. (B) Real-time qPCR analysis of YAP target genes. Apoptosis rate $(C)$, cell viability $(D)$, migratory $(E)$ and invasive ability $(F)$ in the presence of XMU-MP-1, a Mst1/2 inhibitor, also displayed respectively. ${ }^{\star} \mathrm{P}<0.05 ;{ }^{\star *} \mathrm{P}<0.01 ;{ }^{* \star *} \mathrm{P}<0.001$.

\section{Supplementary Files}

This is a list of supplementary files associated with this preprint. Click to download.

- Suppltable1.docx

- supplementarymaterialsandmethods.docx 